\title{
ON THE LOCAL BEHAVIOR OF QUASIREGULAR MAPPINGS
}

\author{
JUKKA SARVAS
}

\section{Introduction}

Consider a non-constant quasiregular mapping $f: G \rightarrow R^{n}$ from a domain $G \subset R^{n}$ into the $n$-dimensional euclidean space $R^{n}, \quad n \geq 2$. Let $B_{f}$ be the branch set of $f$. For any $A \subset R^{n}$ and $y \in R^{n}$ let $N(y, f, A)$ be the cardinality of $A \cap f^{-1}(y)$. We call $N(f, A)=$ $\sup \left\{N(y, f, A) \mid y \in R^{n}\right\}$ the multiplicity of $f$ in $A$. If $i(x, f)$ denotes the local topological index of $f$ at $x \in G$, then

$$
i(x, f)=\min \{N(f, U) \mid U \text { is a neighborhood of } x\} .
$$

Suppose $x \in B_{f}$ and $\Delta$ is an open cone with vertex at $x$ and angle $\alpha \in(0, \pi]$. In $[3,4.4]$ it is proved that if $n \geq 3$ and $\Delta \cap B_{f}=\varnothing$, then $i(x, f) \leq C$, where $C$ is a constant depending only on $n$, $\alpha$ and the maximal dilatation $K(f)$ of $f$. In this paper we will show that if $n \geq 2$ and $f, x$ and $\Delta$ are as above, then $i(x, f) \leq C^{\prime}$, where $C^{\prime}$ is a constant depending only on $n, \alpha, N(f, \Delta)$ and $K(f)$. For $n \geq 3$ we will use this result to derive a new proof for the above cone theorem $[3,4.4]$.

Our notation and terminology is the same as in [2] and [3].

\section{Terminology and preliminary results}

2.1. We let the notation $f: G \rightarrow R^{n}$ include the assumption that $G$ is a domain in $R^{n}$ and $f$ is continuous and non-constant. If $f: G \rightarrow R^{n}$ is quasiregular, we write $K_{I}(f), K_{O}(f)$ and $K(f)$ for the inner, outer and maximal dilatations, respectively.

Suppose that $f: G \rightarrow R^{n}$ is open and discrete. If $x \in G$ and $r>0$, we let $U(x, f, r)$ denote the $x$-component of $f^{-1} B^{n}(f(x), r)$, and for $0<r<d(f(x), \partial f G)$ we write, $l^{*}(x, f, r)=\inf \{|x-y| \mid y \in \partial U(x, f, r)\}$ 
and $L^{*}(x, f, r)=\sup \{|x-y| \mid y \in \partial U(x, f, r)\}$. By [2, 2.9] there exists $r_{x}>0$ such that $U(x, f, r)$ is a normal neighborhood of $x$ for $0<r \leq r_{x}$. If $U\left(x, f, r_{0}\right)$ is a normal neighborhood, it can be shown as in $[2,4.8]$ that the mapping $r \mapsto l^{*}(x, f, r)$ is continuous for $r \in\left(0, r_{0}\right]$.

If $E, F$ and $D$ are subsets of $R^{n}$, we let $\Delta(E, F ; D)$ denote the path family joining $E$ and $F$ in $D$. We say that path family $\Gamma_{1}$ is minorized by path family $\Gamma_{2}$, abbreviated $\Gamma_{1}>\Gamma_{2}$, if every path in $\Gamma_{1}$ has a subpath which is in $\Gamma_{2}$.

If $x \in R^{n}, e \in S^{n-1}$ and $\alpha \in(0, \pi]$, we let Cone $(x, e ; \alpha)$ denote the open infinite cone with vertex at $x$, axis $\{x+t e \mid t>0\}$ and angle $\alpha$, i.e.

$$
\text { Cone }(x, e ; \alpha)=\left\{y \in R^{n}|e \cdot(y-x)>| y-x \mid \cos \alpha\right\},
$$

where $e \cdot(y-x)$ means the scalar product of these vectors. Further we write

$$
b_{n}(\alpha)=m_{n-1}\left(S^{n-1} \cap \text { Cone }(0, e ; \alpha)\right) .
$$

In $[3,5.2]$ it was proved that for a quasiregular mapping $f: G \rightarrow R^{n}$, the inverse linear dilatation $H^{*}(x, f), x \in G$, is bounded in $G$ by a constant depending only on $n$ and $K$. In fact, the proof of $[3,5.2]$ allows a slightly stronger statement. The next lemma is a combined version of this stronger form of $[3,5.2]$ and a part of $[2,2.9]$.

2.3. L e m ma. Let $f: G \rightarrow R^{n}$ be open and discrete, and $x \in G$. Then there exists $r_{x}>0$ such that for every $r \in\left(0, r_{x}\right]$,

(1) $U(x, f, r)$ is a normal neighborhood of $x$,

(2) $\partial U(x, f, r)=U\left(x, f, r_{x}\right) \cap f^{-1} S^{n-1}(f(x), r)$ for $0<r<r_{x}$,

(3) if, in addition, $f$ is $K$-quasiregular, then $L^{*}(x, f, r) \leq C^{*} l^{*}(x, f, r)$, where

$$
C^{*}=C^{*}(n, K)=\exp \left[c_{n} K^{2 /(n-1)}\right],
$$

here $c_{n}$ being a constant depending only on $n$.

Using (3) of the above lemma, we can restate [3,4.3] in a quantitatively better form:

2.5. L e m m a. Suppose that $f: G \rightarrow R^{n}$ is $K$-quasiregular and $x \in G$. Let $r_{x}>0$ and $C^{*}=C^{*}(n, K)$ be as in Lemma 2.3. Then for every $r \in\left(0, r_{x}\right]$, we have: If $|x-y|<l^{*}(x, f, r) / C^{*}$, then

$$
|f(x)-f(y)| \leq r\left(\frac{C^{*}}{l^{*}(x, f, r)}\right)^{\mu}|x-y|^{\mu},
$$

where $\mu=\left(i(x, f) / K_{I}(f)\right)^{1 /(n-1)}$. 
Proof. By Lemma 2.3, for every $r \in\left(0, r_{x}\right], U(x, f, r)$ is a normal neighborhood of $x$ and $L^{*}(x, f, r) / l^{*}(x, f, r) \leq C^{*}$. Fix $r \in\left(0, r_{x}\right]$ and choose $y \in G$ with $0<|y-x|<l^{*}(x, f, r) / C^{*}$. Then we proceed as Martio in $[1,6.1]$. Let $s=|f(x)-f(y)|>0$. Hence,

$$
L^{*}(x, f, s) \leq C^{*} l^{*}(x, f, s) \leq C^{*}|x-y|<l^{*}(x, f, r) .
$$

Thus, $\mathrm{cl} U(x, f, s) \subset B^{n}\left(x, l^{*}(x, f, r)\right)$. For the condenser $(U(x, f, r)$, cl $U(x, f, s))=E$, we have, by [1, 5.13 and 5.15],

$$
\begin{gathered}
\omega_{n-1}\left[\log \frac{r}{s}\right]^{1-n}=\operatorname{cap} f E \leq \frac{K_{I}(f)}{i(x, f)} \operatorname{cap} E \\
\leq \frac{K_{I}(f) \omega_{n-1}}{i(x, f)}\left[\log \frac{l^{*}(x, f, r)}{L^{*}(x, f, s)}\right]^{1-n} .
\end{gathered}
$$

It follows that $s \leq r l^{*}(x, f, r)^{-\mu} L^{*}(x, f, s)^{\mu}$, which implies with (2.6), $|f(x)-f(y)| \leq r l^{*}(x, f, r)^{-\mu} C^{* \mu}|x-y|^{\mu}$. The lemma is proved.

\section{On multiplicity and local index}

3.1. L e m ma. Suppose that $f: G \rightarrow R^{n}$ is $K$-quasiregular and $x \in G$. Let $r_{x}>0$ and $C^{*}=C^{*}(n, K)$ be as in Lemma 2.3. If $m>1, \quad 0<t<L^{*}\left(x, f, r_{x}\right)$ and $A$ is a non-empty Borel set in $U\left(x, f, r_{x}\right)$, then

$$
i(x, f) \leq \omega_{n-1} K_{I}(f) K_{O}(f) \frac{N(f, A)}{M(\Gamma)(\log m)^{n-1}},
$$

where $\Gamma=\Delta\left(S^{n-1}\left(x, t /\left(m C^{* 2}\right)\right), S^{n-1}(x, t) ; A\right)$.

Proof. By $[2,3.2]$,

$$
M(\Gamma) \leq K_{O}(f) N(f, A) M(f \Gamma) .
$$

To derive (3.2) from this inequality, we estimate $M(f \Gamma)$. By Lemma 2.3 and the assumptions of the theorem, $t<L^{*}\left(x, f, r_{x}\right) \leqq C^{*} l^{*}\left(x, f, r_{x}\right)$. Hence, by the continuity of the mapping $r \mapsto l^{*}(x, f, r), 0<r \leq r_{x}$, we can choose $r \in\left(0, r_{x}\right)$ such that $l^{*}(x, f, r)=t / C^{*}$. Set $l^{*}=l^{*}(x, f, r)$. The relation $t /\left(m C^{* 2}\right)=l^{*} /\left(m C^{*}\right)<l^{*} / C^{*}$ implies, by Lemma 2.5, $f S^{n-1}\left(x, t /\left(m C^{* 2}\right)\right) \subset \mathrm{cl} B^{n}\left(f(x), r^{\prime}\right)$, where $r^{\prime}=r\left(C^{*} / l^{*}\right)^{\mu}\left(l^{*} /\left(m C^{*}\right)\right)^{\mu}$ $=r m^{-\mu}$, and $\mu=\left(i(x, f) / K_{I}(f)\right)^{1 /(n-1)}$. Let $\Gamma$ be as in (3.2). Since $L^{*}(x, f, r) \leq C^{*} l^{*}(x, f, r)=t, f \Gamma$ is minorized by

$$
\Gamma^{\prime}=\Delta\left(S^{n-1}\left(f(x), r^{\prime}\right), S^{n-1}(f(x), r) ; B^{n}(f(x), r)\right) .
$$


Then

$$
M(f \Gamma) \leq M\left(\Gamma^{\prime}\right)=\omega_{n-1}\left(\log \left(\frac{r}{r m^{-\mu}}\right)\right)^{1-n}=\omega_{n-1}(\mu \log m)^{1-n} .
$$

Use this upper bound of $M(f \Gamma)$ in (3.3) and the lemma follows.

3.4. Th e o r e m. Suppose that $f: G \rightarrow R^{n}$ is $K$-quasiregular and $x \in G$. Then for every bounded open cone $\Delta=$ Cone $(x, e ; \alpha) \cap B^{n}(x, s)$ with $e \in S^{n-1}, \alpha \in(0, \pi)$ and $s>0$,

$$
N(f, \Delta) \geq \frac{b_{n}(\alpha)}{\omega_{n-1} K_{I}(f) K_{O}(f)} i(x, f),
$$

where $b_{n}(\alpha)$ is as in (2.2).

Proof. Let $r_{x}>0$ be as in Lemma 2.3 and choose $t>0$ so that $0<t<\min \left\{s, l^{*}\left(x, f, r_{x}\right)\right\}$. Set $A=\Delta \cap B^{n}(x, t)$. Then Lemma 3.1 yields for every $m>1$

$$
i(x, f) \leq \omega_{n-1} K_{I}(f) K_{o}(f) \frac{N(f, A)}{M(\Gamma)(\log m)^{n-1}},
$$

where $\quad \Gamma=\Delta\left(S^{n-1}\left(x, t /\left(m C^{* 2}\right)\right), S^{n-1}(x, t) ; A\right)$. By $[4,7.7]$

$$
M(\Gamma)=b_{n}(\alpha)\left[\log \left(m C^{* 2}\right)\right]^{1-n} .
$$

Substitute this in (3.5) and let $m \rightarrow \infty$. The theorem follows because $N(f, A) \leq N(f, \Delta)$.

3.6. Remark. In the plane Theorem 3.4 gives the best possible lower bound for $N(f, \Delta)$. If $k$ and $m$ are positive integers, we define, using the complex notation, $f: C \rightarrow C$ by $f(z)=z^{m k}$ for all $z \in C$, and set

$$
\Delta=\left\{r e^{i \varphi} \mid 0<r<1,0<\varphi<2 \pi / k\right\} .
$$

Then $K(f)=1, i(0, f)=m k$ and $N(f, \Delta)=m$. On the other hand, Theorem 3.4 yields with $\alpha=\pi / k$,

$$
N(f, \Delta) \geq \frac{b_{2}(\pi / k)}{\omega_{1} K(f)^{2}} i(0, f)=\frac{2 \pi / k}{2 \pi \cdot 1} m k=m .
$$

3.7. Open question. Theorem 3.4 implies that there exists $z_{0} \in \Delta$ such that $f$ assumes the value $f\left(z_{0}\right)$ at least $b_{n}(\alpha)\left(\omega_{n-1} K^{2}\right)^{-1} i(x, f)$ times in $\Delta$. Is this typical of all $z \in \Delta$ (which are sufficiently close to $x$ )? The question could be formulated explicitly as follows: If $f$ and $\Delta$ are as in Theorem 3.4 with $m \geq 1$ such that there are points $y \in \Delta$ arbitrarily close to $x$ with $N(y, f, \Delta) \leq m$, is $i(x, f)$ then bounded by some constant depending only on $n, K, \alpha$ and $m$ ? 


\section{On the local structure of $B_{f}$}

4.1. In this section we want to show that the cone theorem $[3,4.4]$ can be derived from Theorem 3.4, too. Thus Theorem 3.4 can be considered as a generalization of $[3,4.4]$. First we need the following lemma.

4.2. L e m ma. Suppose that $f: G \rightarrow R^{n}$ is $K$-quasiregular and $x \in G$. Let $r_{x}>0$ and $C^{*}=C^{*}(n, K)$ be as in Lemma 2.3. If $A \subset U\left(x, f, r_{x}\right)$, then

$$
N(f, A)=\max _{t>0} N\left(f, A \cap\left[\operatorname{cl} B^{n}(x, t) \backslash B^{n}\left(x, t / C^{*}\right)\right]\right) .
$$

Proof. Choose any $y \in A \subset U\left(x, f, r_{x}\right)$. By Lemma 2.3 for $s=|f(x)-f(y)|, \quad \partial U(x, f, s)=U\left(x, f, r_{x}\right) \cap f^{-1} S^{n-1}(f(x), s) \quad$ and $L^{*}(x, f, s) \leq C^{*} l^{*}(x, f, s)$. Then for $t=L^{*}(x, f, s)$ we have

$A \cap f^{-1}(f(y)) \subset A \cap \partial U(x, f, s) \subset A \cap\left[\operatorname{cl} B^{n}(x, t) \backslash B^{n}\left(x, t / C^{*}\right)\right]$.

The lemma follows.

4.3. Th e o r e m $[3,4.4]$. Suppose that $f: G \rightarrow R^{n}$ is $K$-quasiregular, $n \geq 3$ and $x \in G$. If

$$
\text { Cone }(x, e ; \alpha) \cap B^{n}(x, t) \subset G \backslash B_{f}
$$

with $e \in S^{n-1}, \alpha \in(0, \pi / 2)$ and $t>0$, then $i(x, f) \leq C$, where $C$ depends only on $n, K$ and $\alpha$.

Proof. Let $C^{*}=C^{*}(n, K)$ and $r_{x}>0$ be as in Lemma 2.3. Set $s_{0}=\min \left\{l^{*}\left(x, f, r_{x}\right), t / 2\right\}$. Let $\psi=\psi(n, K)$ be the constant in $[3,2.3]$. Then by $[3,2.7] f$ is injective in every ball $B^{n}(x+s e, \gamma s)$ with

$$
\gamma=\psi \sin \alpha, 0<s \leq s_{0} .
$$

Define balls $B_{i}=B^{n}\left(x_{i}, r_{i}\right), i=1,2, \ldots$, by setting

$$
\begin{aligned}
& x_{1}=x+s_{0} e, \quad r_{1}=\gamma\left|x_{1}-x\right| \text { and } \\
& x_{i}=x_{i-1}-r_{i-1} e, \quad r_{i}=\gamma\left|x_{i}-x\right|, \quad i>1 .
\end{aligned}
$$

Then $f$ is injective in every $B_{i}$ and it is easy to see that $\cup_{i} B_{i}$ covers the set

$$
\Delta_{\beta}=\text { Cone }(x, e ; \beta) \cap B^{n}\left(x, s_{0}\right),
$$

where $\beta \in(0, \pi / 2)$ with $\tan \beta=\gamma / 3^{1 / 2}$. So $\beta$ depends only on $n, K$ and $\alpha$. We want to show that $N\left(f, \Delta_{\beta}\right)$ has an upper bound depending only on $n, K$ and $\alpha$, which will prove the theorem by Theorem 3.4 .

To estimate $N\left(f, \Delta_{\beta}\right)$ we apply Lemma 4.2. Consider any $s \in\left(0, s_{0}\right]$. It is easy to see that to cover the set

$$
\Delta_{\beta}^{s}=\Delta_{\beta} \cap\left[\operatorname{cl} B^{n}(x, s) \backslash B^{n}\left(x, s / C^{*}\right)\right]
$$


with balls $B_{i}$ we need at most $m_{s}$ balls, where $m_{s}$ is the smallest integer greater than $\left(s-s / C^{*}\right) /\left(\gamma s / C^{*}\right)+1=1+\left(C^{*}-1\right) / \gamma$. Hence $N\left(f, \Delta_{\beta}^{s}\right) \leq 2+\left(C^{*}-1\right) / \gamma$. Since $s \in\left(0, s_{0}\right]$ is arbitrary, Lemma 4.2 implies $N\left(f, \Delta_{\beta}\right) \leqq 2+\left(C^{*}-1\right) / \gamma$. This upper bound of $N\left(f, \Delta_{\beta}\right)$ depends only on $n, K$ and $\alpha$. The theorem is proved.

\section{References}

[1] Martio, O.: A capacity inequality for quasiregular mappings. - Ann. Acad. Sci. Fenn. Ser. A I 474, 1970, 1-18.

[2] MaRTIO, O., S. RICKMAN, and J. VÄISÄL ̈̈: Definitions for quasiregular mappings. - Ann. Acad. Sci. Fenn. Ser. A I 448, 1969, 1-40.

[3] -»- Topological and metric properties of quasiregular mappings. - Ann. Acad. Sci. Fenn. Ser. A I 488, 1971, 1-31.

[4] VÄISÄL Notes in Mathematics 229, Springer-Verlag, Berlin-Heidelberg-New York, 1971.

University of Helsinki

Department of Mathematics

SF-00100 Helsinki 10

Finland

Received 21 February 1975 